\title{
Intervallfasten führt in der Altersgruppe „45-Plus“ zu einer Abnahme von Körpergewicht und Bauchumfang
}

\section{EINLEITUNG}

Regelmäßigen täglichen Esspausen von mindestens 16 Stunden werden positive Effekte auf Stoffwechsel, Körpergewicht und Allgemeinbefinden nachgesagt.

\section{FRAGESTELLUNG}

Unter jungen Erwachsenen ist das Intervallfasten nach der 16:8-Methode sehr populär. Inwieweit sich das Ernährungskonzept auch für ältere Menschen eignet, ist bisher nicht untersucht.

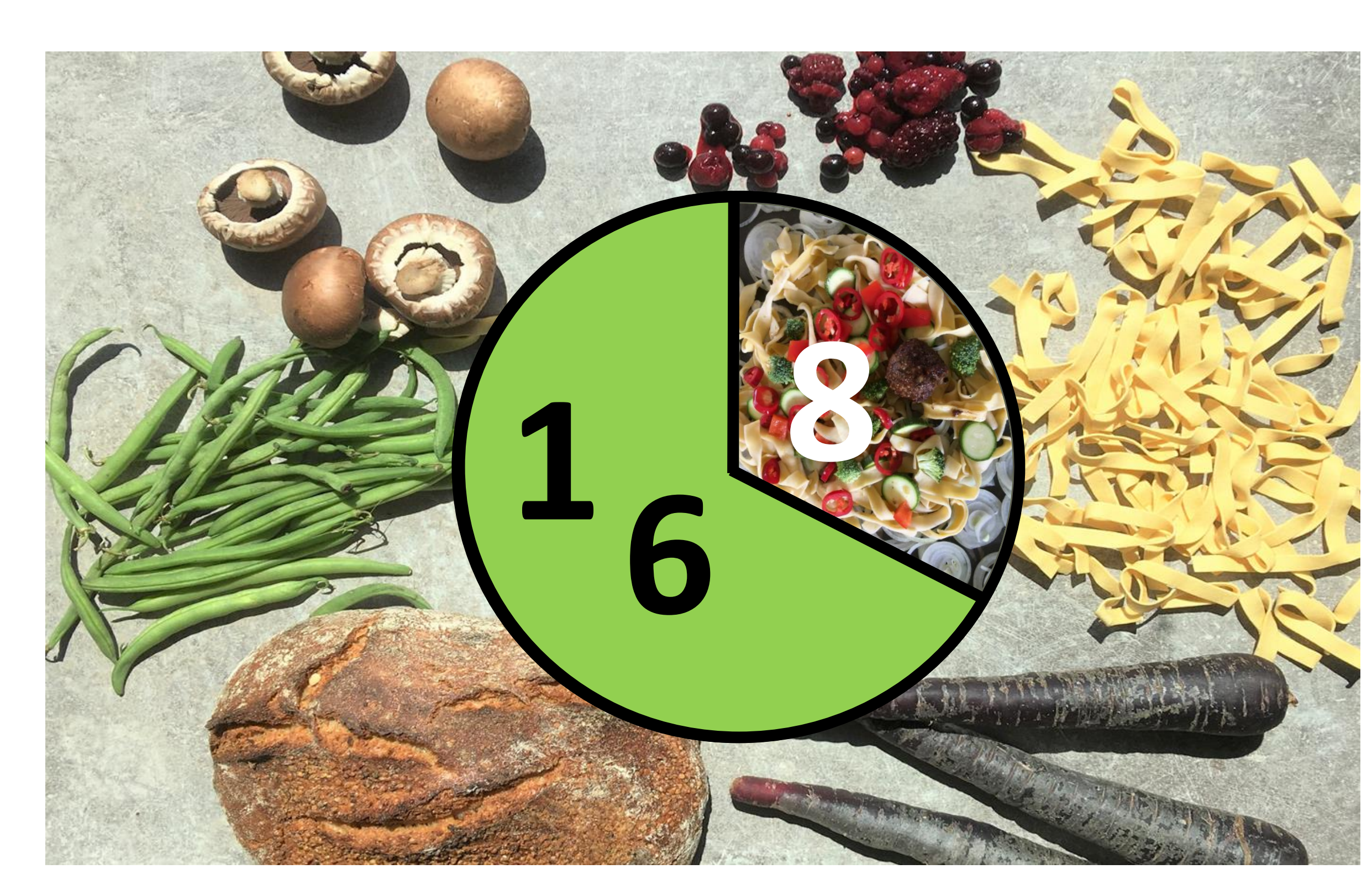

\section{Gewichtsabnahme}

Nach vier Wochen hatten 66 von 74 Teilnehmern*innen Gewicht verloren (89,2\%):

- Frauen 1,4 kg; Männer 1,9 kg (Median)

- drei Frauen zeigten keine Veränderung

- drei Frauen zeigten eine Zunahme

Insgesamt nahmen 37 Teilnehmer*innen (50\%) zwischen 1,5 und $6 \mathrm{~kg}$ ab.

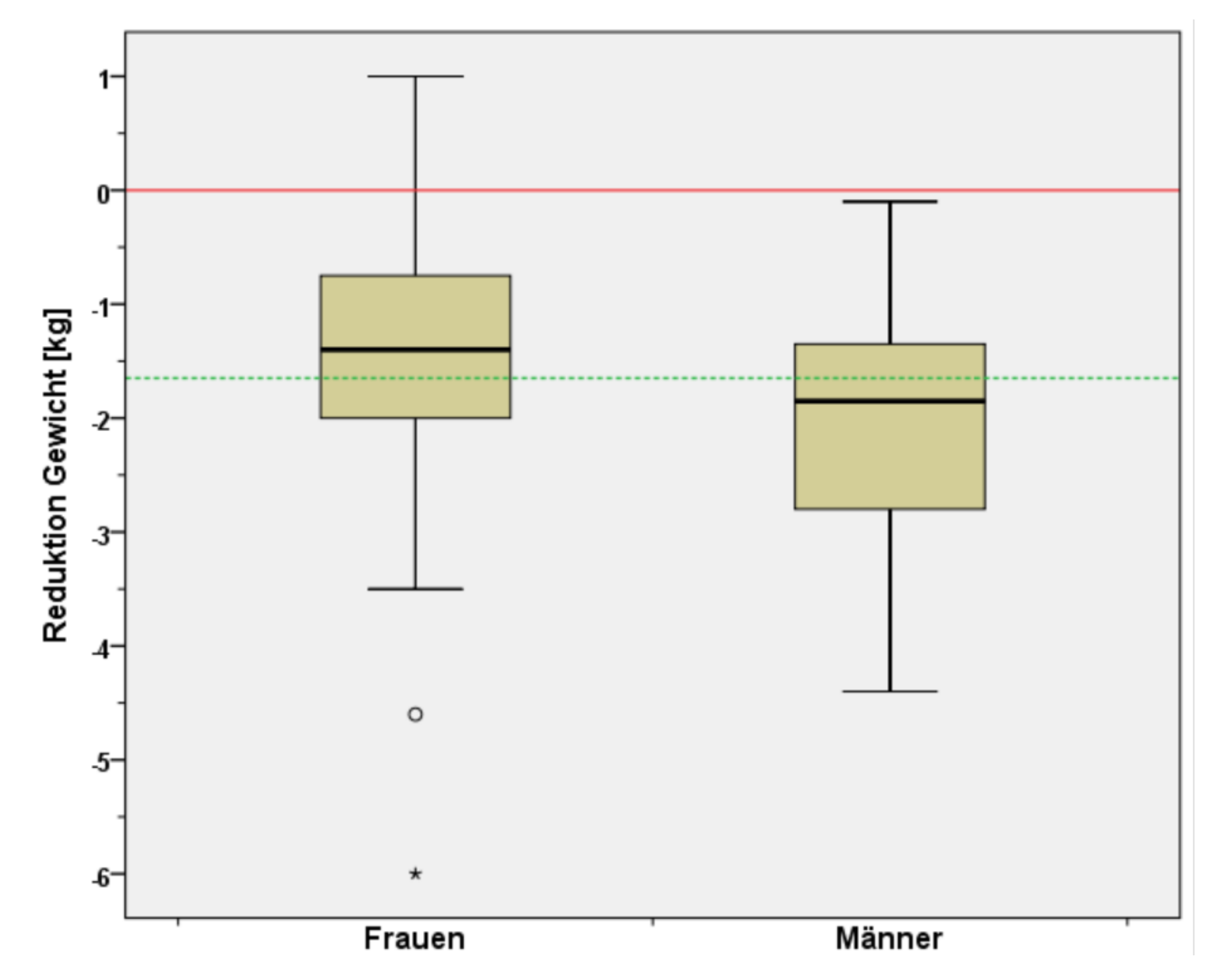

\section{METHODE}

Untersucht wurde die Wirksamkeit der 16:8-Methode über vier Wochen unter Real-Life-Bedingungen bei 45 bis 78-Jährigen im Rahmen eines Vorher-Nachher-Vergleichs. Eingeschlossen wurden Probanden*innen mit einem BMI $\geq 18,5 \mathrm{~kg} / \mathrm{m}^{2}$.

\section{Studiencharakteristika}

\begin{tabular}{|l|l|}
\hline Intervention & Intervallfasten nach der 16:8-Methode \\
\hline Studiendesign & Vorher-Nachher-Vergleich (ohne Kontrollgruppe) \\
\hline Primäre Endpunkte & Reduktion von Gewicht und Bauchumfang \\
\hline Beobachtungsdauer & 4 Wochen \\
\hline Auswertung & deskriptiv \\
\hline Rekrutierte Probanden & $\mathrm{N}=89$ \\
\hline Rücklautverluste (lost to follow-up) & $\mathrm{N}=12(13,5 \%)$ \\
\hline Rücklaufquote & $\mathrm{N}=77(86,5 \%)$ \\
\hline Ausgeschlossene Teilnehmer & $\mathrm{N}=3$ (Verletzung der Alters-Einschlusskriterien) \\
\hline Auswertbare Teilnehmer & $\mathrm{N}=74$ (inklusive zwei Studienabbrecher) \\
\hline
\end{tabular}

\section{Teilnehmercharakteristika}

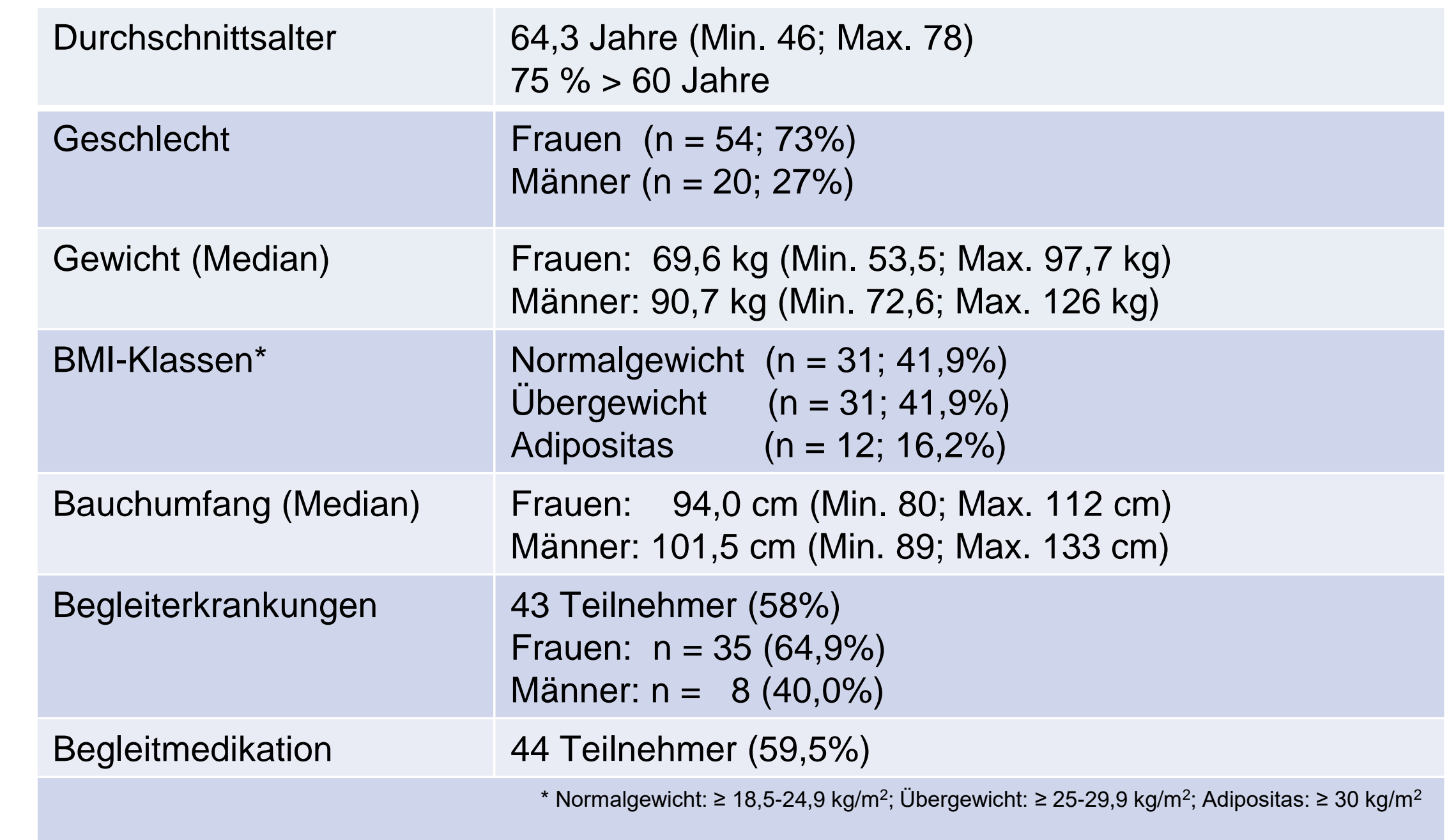

\section{Adhärenz und Persistenz}

\section{Verträglichkeit}

Die Vitalparameter (Ruhepuls, Blutdruck, allgemeines Wohlbefinden) zeigten keine relevanten Veränderungen. Die Hälfte aller Teilnehmer*innen $(51,4 \%)$ berichtete über temporäre Befindlichkeitsstörungen. Zwei Probanden*innen (2,7\%) haben die Pilotstudie aus medizinischen Gründen (Blutdruckabfall, Gastritis) abgebrochen.

\begin{tabular}{|c|c|c|}
\hline Parameter (Mediane) & Vorher & Nachher \\
\hline $\begin{array}{l}\text { Allgemeines Wohlbefinden } \\
0=\text { schlechtester, } 10=\text { bester Wert }\end{array}$ & $\begin{array}{l}\text { Frauen: } 7,0 \\
\text { Männer: } 7,5\end{array}$ & $\begin{array}{l}\text { Frauen: } 7,1 \\
\text { Männer: } 7,6\end{array}$ \\
\hline Ruhepuls (Schläge/Minute) & $\begin{array}{l}\text { Frauen: } 67,5 \\
\text { Männer: } 68,0\end{array}$ & $\begin{array}{l}\text { Frauen: } 69 \\
\text { Männer: } 66\end{array}$ \\
\hline $\begin{array}{l}\text { Blutdruck }(\mathrm{mmHg}) \\
(\mathrm{n}=45 \text { Probanden*innen) }\end{array}$ & $\begin{array}{l}\text { Frauen: } 129 / 79 \\
\text { Männer 125/79 }\end{array}$ & $\begin{array}{l}\text { Frauen: } 126 / 78 \\
\text { Männer: } 125 / 78\end{array}$ \\
\hline
\end{tabular}

Autoren und Institutionen

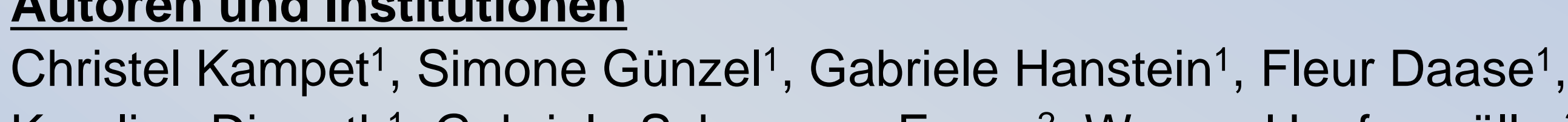
Karoline Dimroth ${ }^{1}$, Gabriele Schaepers-Feese ${ }^{2}$, Werner Hopfenmüller ${ }^{3}$ Franz-Werner Dippel ${ }^{1}$

Studierende des BANA-Gasthörerstudiums, Schwerpunkt Ernährung \& Gesundheit, Technische Universität Berlin. Zentraleinrichtung Wissenschaftliche Weiterbildung und Kooperation (ZEWK),

Technische Universität Berlin.
3 Institut für Biometrie und Klinische Epidemiologie, Charité, Berlin.
Die Fasten- bzw. Essintervalle (von 16:8 Std.) wurden an $86 \%$ aller Studientage eingehalten.

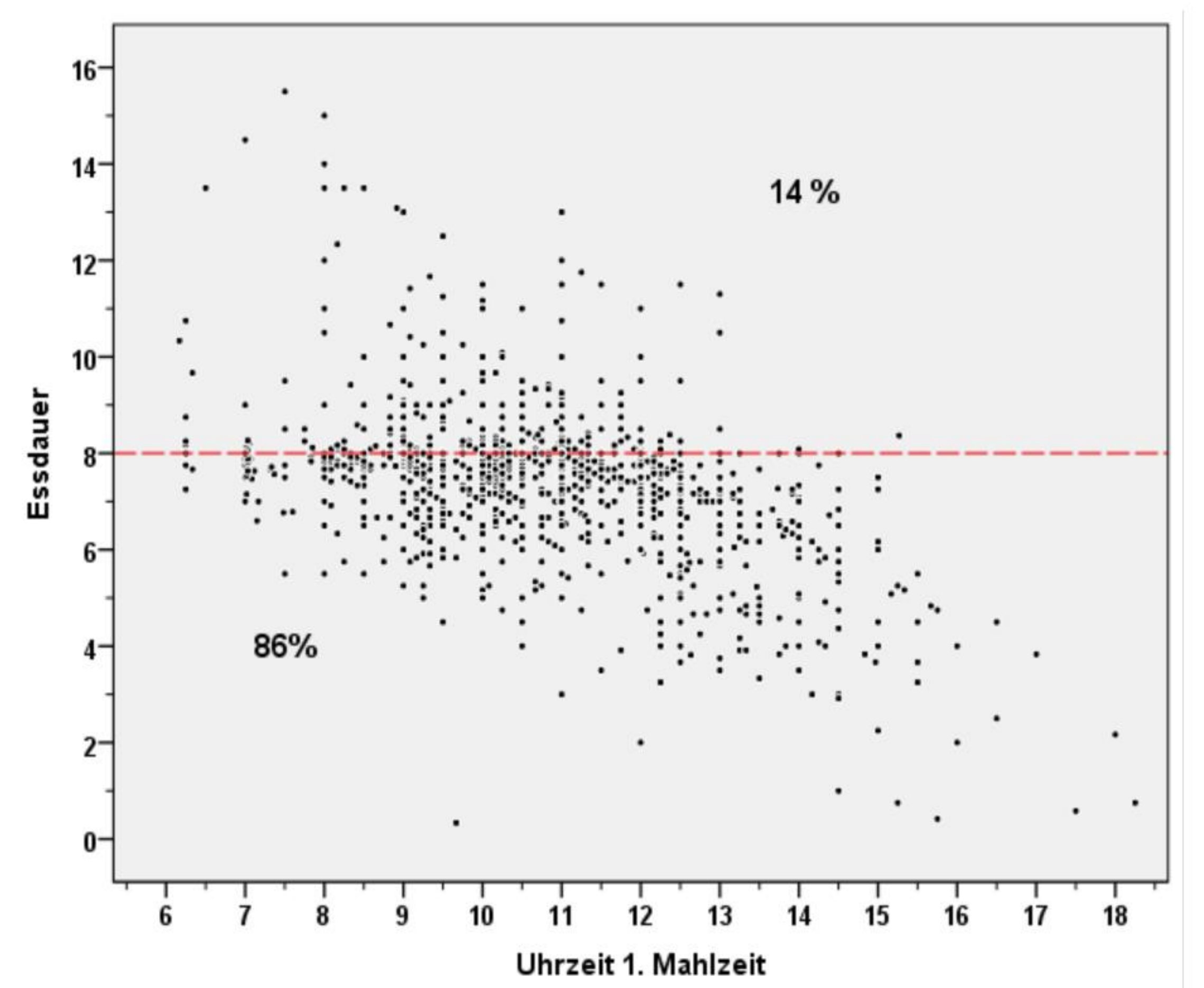

Zeitschrift: Ernährung \& Medizin DOI: $10.1055 / a-1115-9709$

\section{Abnahme des Bauchumfangs}

Nach vier Wochen hatte sich der Bauchumfang bei 58 von 74 Teilnehmern*innen verringert $(78,4 \%)$ :

- Frauen $2 \mathrm{~cm}$; Männer 2,3 cm (Median)

- zwölf Probanden*innen zeigten keine Veränderung - zwei weitere zeigten eine Zunahme Bei 45 Probanden*innen $(61 \%)$ verringerte sich der Bauchumfang um mehr als $2 \mathrm{~cm}$.

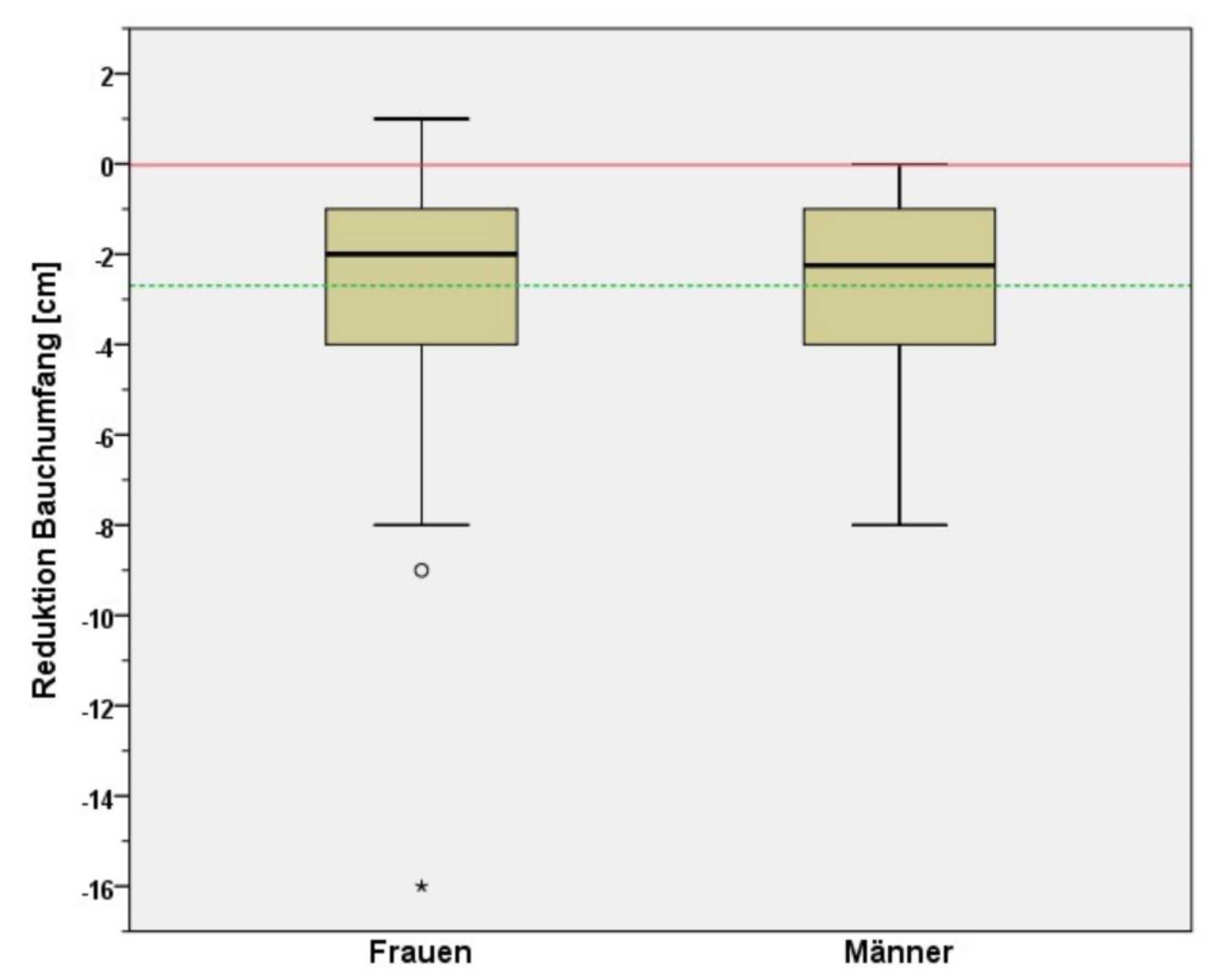

\section{ZUSAMMENFASSUNG}

- Pilotstudie bei 45 bis 78-Jährigen

- 16-stündige Fastenintervalle täglich

- über 4 Wochen

- unter Real-Life-Bedingungen

- Gewicht: $-1,4 \mathrm{~kg}(\$) ;-1,9 \mathrm{~kg}($ ( $)$

- Bauchumfang: $-2,0 \mathrm{~cm}(\&) ;-2,3 \mathrm{~cm}$ (ळ)

- Allg. Gesundheitszustand: unverändert

- Integration in den Alltag: einfach

\section{FAZIT}

Eine Gewichtsreduktion von 1,5 bis $2 \mathrm{~kg} /$ Monat gilt gemäß DAG-Leitlinie als relevanter Abnehmerfolg. Das Ergebnis erfüllt somit die allg. Anforderungen an eine erfolgreiche Ernährungsintervention.
36. Jahrestagung der Deutschen Adipositas-Gesellschaft (DAG) e. V., 08.-10. Oktober 2020, Leipzig. Korrespondenzadresse: f.dippel@campus.tu-berlin.de 